\title{
Pesquisa clínica com o Toque Terapêutico: uma revisão sistemática
}

\author{
Clinical research with Therapeutic Touch: a systematic review
}

\author{
André Luiz Thomaz de Souza ${ }^{1}$, Bárbara de Oliveira Prado', Roberta de Paiva Silva² \\ Érika de Cassia Lopes Chaves ${ }^{3}$, Denise Hollanda lunes ${ }^{4}$ \\ ${ }^{1}$ Mestrandos em Enfermagem pela Universidade Federal de Alfenas (UNIFAL), Alfenas, MG. \\ ${ }^{2}$ Mestre em Enfermagem pela UNIFAL, Alfenas, MG. \\ ${ }^{3}$ Doutora em Enfermagem. Professora na Escola de Enfermagem da UNIFAL, Alfenas, MG \\ ${ }^{4}$ Doutora em Fisioterapia. Professora no Curso de Fisioterapia da UNIFAL, Alfenas, MG.
}

\section{RESUMO}

Objetivos: Investigar os protocolos utilizados em pesquisas clínicas sobre a modalidade de terapia complementar Toque Terapêutico, especificamente perfil do público alvo, número, tempo e frequência das sessões, bem como as principais evidências encontradas.

Fonte de Dados: A investigação foi realizada nas bases de dados Medline/PubMed, Lilacs e Biblioteca Cochrane, selecionando estudos com metodologia experimental, publicados no período de 2004 e 2014, nos idiomas português e inglês, por meio dos descritores Toque Terapêutico (Therapeutic Touch), Enfermagem (Nursing) e Terapia Alternativa/Complementar (Alternative/Complementary Therapy).

Síntese dos Dados: Dentre os oito estudos selecionados, observa-se que as sessões aplicadas variam de três a 28, com duração média entre 15 e 90 minutos. O público alvo vai desde indivíduos saudáveis até pessoas hospitalizadas com queimaduras, em que a maioria dos artigos retrata uma melhora significativa no escore de dor e ansiedade, bem como nos níveis de hemoglobina e hematócrito.

Conclusão: Os artigos demonstraram efeitos positivos quanto ao uso do Toque Terapêutico nas pesquisas clinicas. Entretanto, pela ausência de uma padronização no seu uso, são encontradas dificuldades na replicação dos métodos já estudados e, consequentemente, na validação de um padrão que possa ser utilizado na prática e em outras pesquisas.

DESCRITORES: TOQUE TERAPÊUTICO; ENFERMAGEM; TERAPIAS COMPLEMENTARES; TERAPIAS ALTERNATIVAS.

\section{ABSTRACT}

Aims: To investigate clinical research protocols using the Therapeutic Touch modality of complementary therapy, identifying the profile target audiences as well as the number, time and frequency of sessions.

Source of Data: The investigation was performed in the Medline/PubMed, Lilacs and Cochrane Library databases, selecting studies with experimental methodology, published between 2004 and 2014, in Portuguese and English languages, through the terms Therapeutic Touch, Nursing and Alternative/Complementary Therapy.

Summary of Findings: Among the eight selected studies, the number of sessions ranged from three to 28, and the average time per session was between 15 and 90 minutes. The target audience ranged from healthy subjects to persons hospitalized in burn units. Most of the articles reported a significant improvement in pain scores and anxiety, as well as in hemoglobin and hematocrit.

Conclusions: The reviewed articles demonstrated positive effects on the use of Therapeutic Touch in clinical research. However, because of the lack of standardization in its use, difficulties in replicating the studied methods and, therefore, in the validity of a pattern that could be used in clinical practice as well as in research.

KEY WORDS: THERAPEUTIC TOUCH; NURSING; COMPLEMENTARY THERAPIES. 


\section{INTRODUÇÃO}

Nas últimas décadas o uso da medicina complementar tem sido recorrente no tratamento de diversas condições patológicas e nos mais variados grupos de pacientes. ${ }^{1-3}$ Nos Estados Unidos, cerca de $40 \%$ da população adulta tem utilizado alguma forma dessas terapias, sendo que em 2007 a população gastou em torno de 34.000 milhões de dólares com uso de terapias complementares e/ou alternativas. ${ }^{4}$

Entretanto, é notória a demanda por estudos primários e secundários que envolvam o uso das terapias complementares. Para Thiago e Tesser, ${ }^{5}$ é fundamental o envolvimento dos profissionais da saúde com essas modalidades de intervenção, principalmente por se tratarem de intervenções que possibilitam a desmedicalização do cuidado, além de terem boa aceitação social. No Brasil, ressalta-se como passo importante para uma nova perspectiva de atendimento o princípio doutrinário de integralidade do Sistema Único de Saúde, formalizado na oitava Conferencia Nacional de Saúde, na qual torna relevante a oferta de terapias complementares nos serviços de saúde. ${ }^{6}$

No intuito de garantir essa integralidade no atendimento à saúde, a Política Nacional de Medicina Natural e Práticas Complementares (PMNPC) ${ }^{6}$ do Sistema Único de Saúde (SUS) apoia o uso de terapias nessas modalidades, com o interesse de atuar na prevenção de agravos e na promoção, manutenção e recuperação da saúde. Destacam-se nessa política a acupuntura, a homeopatia, a fitoterapia e a medicina antroposófica. Por outro lado, o Toque Terapêutico, uma modalidade de terapia complementar que, segundo a literatura científica, ${ }^{7,89}$ apresenta efetividade significativa sobre o tratamento de diversas doenças, não faz parte da PMNPC, embora nas últimas décadas venha ocorrendo um crescente interesse na investigação científica evolvendo o uso desta modalidade de terapia complementar. ${ }^{10}$

O Toque Terapêutico é uma versão contemporânea de antigas práticas de cura por meio da imposição de mãos sobre o campo energético humano (CEH), que tem como ponto de referência para o início de sua utilização os estudos desenvolvidos por Dolores Krieger e Dora Kunz na década de 1970. ${ }^{11}$ Essa modalidade de intervenção faz parte dos conceitos da medicina vibracional, a qual considera o corpo como um sistema dinâmico de energia em que a mente e o espírito são fontes da consciência e detêm o poder de ocasionar doenças por meio de interações energéticas e neuro-hormonais nas dimensões do corpo, da mente e do espírito, presumindo que intervenções sobre no CEH possam provocar efeitos na saúde do indivíduo. ${ }^{12} \mathrm{O} \mathrm{CEH}$ é definido como uma junção de componentes eletrostáticos, magnéticos, térmicos e visuais, harmonizados por meio do processo fisiológico normal, que se extrapola além do organismo, tornandose concentrado ao redor de seres vivos. ${ }^{13}$ Desse modo, o Toque Terapêutico, segundo Krieger, ${ }^{11}$ consiste no uso consciente da imposição de mãos como forma de modular e de reorganizar o $\mathrm{CEH}$, quando este se encontra em desequilíbrio.

De acordo com o método Krieger-Kunz, o Toque Terapêutico envolve basicamente quatro etapas na sua aplicação, que devem ser cumpridas na seguinte ordem: centralização da consciência (passo inicial onde o terapeuta focaliza sua atenção na sensibilidade das mãos, concentrando suas energias para realização do diagnóstico do $\mathrm{CEH}$ ); avaliação do campo de energia (etapa que consiste em tocar o $\mathrm{CEH}$ percebendo suas anormalidades, assimetrias, calor, frio, bloqueios, choques, formigamentos); reequilíbrio ou repadronização do $\mathrm{CEH}$ (momento onde o terapeuta realiza a imposição das mãos com objetivo de reestabelecer os padrões de normalidade); e reavaliação do $\mathrm{CEH}$ (consiste em verificar novamente o $\mathrm{CEH}$, realizando comparações com o que foi encontrado na segunda etapa). ${ }^{11,14,15}$

Um ponto importante a ser considerado é que o Toque Terapêutico se revela como um excelente meio não invasivo de que o enfermeiro e outros profissionais lançam mão para o tratamento de diversas doenças. ${ }^{14,16}$ Além de ser uma estratégia de cuidado que pode ser utilizada pela enfermagem no tratamento da dor, no alívio da ansiedade, na cicatrização da pele e no estresse, pode ser utilizado também em situações onde ocorra um desequilíbrio no CEH. ${ }^{11,17}$ Entretanto, percebe-se que um número reduzido de profissionais, no Brasil, domina essa técnica. ${ }^{18}$

Considerando as muitas limitações encontradas na literatura quanto aos grupos específicos de pacientes, número de intervenções, tempo de cada intervenção e, por outro lado, o fato de ser uma técnica de baixo custo e em destaque no meio científico, acredita-se na importância da investigação científica por meio de estudos que possam estabelecer métodos reprodutíveis em diferentes condições. Deste modo, objetivou-se, nesta revisão, investigar os protocolos utilizados no Toque Terapêutico em pesquisas clínicas.

\section{METODOLOGIA}

Este estudo consiste em uma revisão sistemática elaborada de acordo com os critérios estabelecidos 
pelo PRISMA Statement (Preferred Reporting Items for Systematic Reviews and Meta-Analyses), ${ }^{19}$ instrumento que auxilia os autores na estruturação adequada de uma revisão sistemática ou de metanálises no intuito de garantir um estudo secundário transparente. ${ }^{20}$

A busca pelos estudos foi realizada nos meses de janeiro e fevereiro de 2014 por meio dos descritores: Toque Terapêutico (Therapeutic Touch), Enfermagem (Nursing) e Terapias Alternativas/Complementares (Alternative/Complementary Therapies) consultados nos Descritores em Ciência da Saúde (DeCS) e nos Medical Subject Headings (MeSH). A investigação fundamentou-se nos estudos encontrados nas bases de dados Pubmed/Medline, Lilacs e Biblioteca Cochrane. Foram incluídos na revisão apenas estudos realizados com humanos, com metodologia baseada em estudos experimentais, disponíveis na íntegra e de livre acesso nos idiomas português e inglês, publicados entre o período de 2004 e 2014.

Os estudos selecionados deveriam obrigatoriamente responder ao seguinte questionamento: "como tem sido o uso do Toque Terapêutico nas pesquisas?" Especificamente buscou-se identificar o perfil do público alvo, o número, o tempo e a frequência das sessões, bem como as principais evidências encontradas. Frente aos critérios de elegibilidade estabelecidos, excluíram-se revisões da literatura e artigos que não respondessem às questões norteadoras.

Após as buscas, foi realizada por dois pesquisadores uma análise criteriosa do material disponível por meio da leitura exploratória, seletiva, analítica e interpretativa. ${ }^{21}$ Após a primeira seleção dos estudos, os artigos que potencialmente responderiam às questões foram avaliados pelos mesmos pesquisadores e, posteriormente, foram confrontados os dados encontrados. Para tanto, os estudos foram considerados adequados em função do alocamento dos grupos experimentais e de controle e das informações que respondessem a esta revisão. Os artigos que não preenchessem esses critérios foram excluídos. $\mathrm{Na}$ síntese dos dados foi utilizado um formulário elaborado pelos autores, o qual contemplava as seguintes informações: o número, o tempo e a frequência das sessões, bem como as principais evidências encontradas.

\section{RESULTADOS DA SELEÇÃO DOS ARTIGOS}

Identificou-se um total de 545 artigos, distribuídos do seguinte modo: 455 na base de dados Medline, 37 na Lilacs e 58 na Cochrane. Foram selecionados para leitura dos resumos e títulos 38 artigos, sendo estes
32 na Medline, um na Lilacs, e cinco na Cochrane, sendo excluídos desse montante 30 artigos, por motivos relacionados à repetição nas bases de dados, metodologia utilizada, ano de publicação e tipo de terapia alternativa adotada. Ao final, foram selecionados para esta revisão oito artigos (Figura 1).

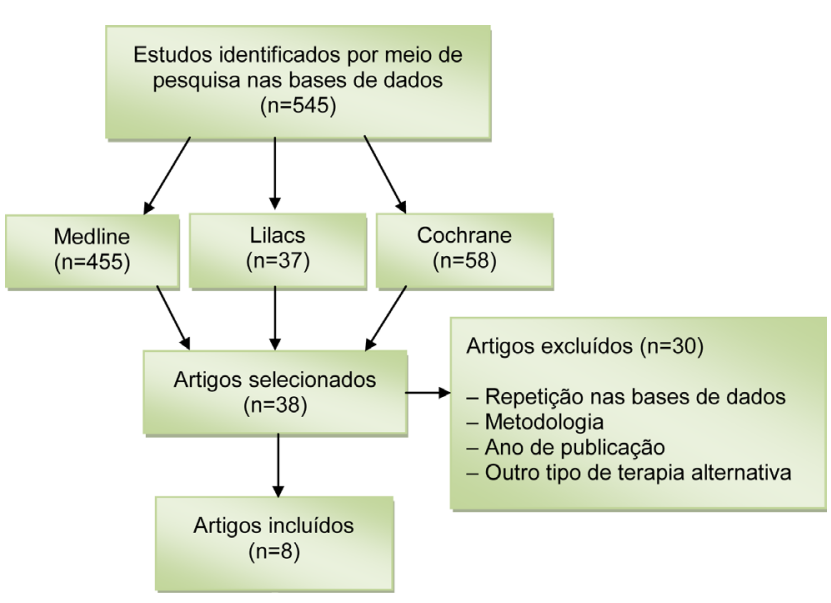

Figura 1. Fluxograma dos estudos selecionados sobre Toque Terapêutico

\section{CONTEÚDO DA REVISÃO}

Dentre os oito estudos selecionados, observou-se que apenas um foi desenvolvido no Brasil. A população de estudo apresentava características distintas, desde pessoas com doença de Alzheimer a indivíduos sadios (Tabela 1).

No que se refere ao número de sessões de Toque Terapêutico, as intervenções variam de três a 28 sessões. Quanto à duração das sessões, os estudos avaliados apresentavam diversas situações, com tempo de sessão variando entre 10 a 90 minutos. Com relação à frequência com que eram realizadas as intervenções, ocorreram sessões em um único dia, em três etapas, e até sessões realizadas diariamente durante uma semana.

Dentre os principais resultados encontrados, destacam-se a melhora significativa na dor, no sono e na ansiedade, verificadas na maioria dos estudos. Entretanto, um dos estudos avaliados não apresentou resultados estatísticos favoráveis ao uso do Toque Terapêutico. Os autores apresentam como fator limitante o tamanho reduzido da amostra, destacando que as investigações sobre terapias na prática de enfermagem devem ser realizadas por enfermeiros com experiência em pesquisa. ${ }^{9}$ As demais características pertinentes aos estudos estão relacionadas na Tabela 2 . 
Tabela 1. Revisão sistemática sobre Toque Terapêutico - estudos selecionados

\begin{tabular}{|c|c|c|c|c|}
\hline Autores & Ano & País & Amostra & Finalidade do tratamento \\
\hline Busch et al. ${ }^{9}$ & 2012 & Holanda & 19 pessoas & $\begin{array}{l}\text { Avaliar o efeito do Toque Terapêutico na redução da ansiedade, } \\
\text { dor, níveis de cortisol e medicações utilizadas para dor, em pessoas } \\
\text { hospitalizadas com queimadura }\end{array}$ \\
\hline Marta et al..$^{22}$ & 2010 & Brasil & 30 pessoas & $\begin{array}{l}\text { Avaliar os efeitos do Toque Terapêutico na diminuição da ansiedade, } \\
\text { no escore de dor e depressão, e na melhorara o sono em pessoas com } \\
\text { dor crônica não oncológica }\end{array}$ \\
\hline Aghabati et al. ${ }^{23}$ & 2010 & Irã & 90 pessoas & $\begin{array}{l}\text { Avaliar os efeitos do Toque Terapêutico no controle da dor e fadiga } \\
\text { em pacientes submetidos ao tratamento quimioterápico }\end{array}$ \\
\hline Hawranik et al. ${ }^{24}$ & 2008 & Canadá & 51 pessoas & $\begin{array}{l}\text { Comparar a eficácia do Toque Terapêutico com o toque simulado nos } \\
\text { sintomas de agressão não física e agitação verbal em pessoas } \\
\text { com Alzheimer }\end{array}$ \\
\hline Maclntyre et al..$^{25}$ & 2008 & Estados Unidos & 120 pessoas & $\begin{array}{l}\text { Avaliar os efeitos do Toque Terapêutico na diminuição do uso de } \\
\text { medicamentos, tempo de internação, incidência de fibrilação cardíaca e } \\
\text { condições do escore de ansiedade em pessoas submetidas à cirurgia de } \\
\text { revascularização do miocárdio }\end{array}$ \\
\hline Wang et al. ${ }^{26}$ & 2006 & Estados Unidos & 14 pessoas & $\begin{array}{l}\text { Avaliar os efeitos do Toque Terapêutico nos níveis de agitação em pessoas } \\
\text { com demência }\end{array}$ \\
\hline Movaffaghi et al. ${ }^{16}$ & 2006 & Irã & 86 pessoas & $\begin{array}{l}\text { Investigar os efeitos do Toque Terapêutico nos níveis de hemoglobina } \\
\text { e hematócrito em estudantes saudáveis }\end{array}$ \\
\hline Larden et al. ${ }^{27}$ & 2004 & Canadá & 54 pessoas & $\begin{array}{l}\text { Investigar se o Toque Terapêutico em mulheres sob tratamento de } \\
\text { dependência influencia nos níveis de ansiedade e abstinência }\end{array}$ \\
\hline
\end{tabular}

Tabela 2. Método utilizado para o desenvolvimento do Toque Terapêutico

\begin{tabular}{|c|c|c|c|c|}
\hline Autores & $\begin{array}{l}\text { Característica da } \\
\text { população estudada }\end{array}$ & $\begin{array}{l}\text { Número de } \\
\text { sessões }\end{array}$ & $\begin{array}{l}\text { Tempo e frequência das } \\
\text { sessões }\end{array}$ & Principais resultados encontrados \\
\hline Busch et al. ${ }^{9}$ & $\begin{array}{l}\text { Pessoas hospitalizadas com } \\
\text { queimadura }\end{array}$ & 10 & $\begin{array}{l}\text { Uma vez ao dia durante } 10 \\
\text { dias com duração média de } \\
5 \text { a } 15 \text { minutos cada sessão }\end{array}$ & $\begin{array}{l}\text { Descrição dos dados no artigo menciona baixo } \\
\text { poder estático dos resultados encontrados }\end{array}$ \\
\hline Marta et al..$^{22}$ & $\begin{array}{l}\text { Pessoas com dor crônica } \\
\text { não oncológica }\end{array}$ & 8 & $\begin{array}{l}\text { Duas vezes por semana } \\
\text { com duração de } 25 \text { minutos } \\
\text { cada sessão }\end{array}$ & $\begin{array}{l}\text { Melhora significativa na intensidade de dor, na } \\
\text { avaliação da depressão e na qualidade do sono }\end{array}$ \\
\hline Aghabati et al. ${ }^{23}$ & $\begin{array}{l}\text { Pessoas com câncer } \\
\text { apresentando sintomas de } \\
\text { dor e fadiga }\end{array}$ & 5 & $\begin{array}{l}\text { Uma vez ao dia durante } \\
5 \text { dias com duração de } \\
30 \text { minutos cada sessão }\end{array}$ & $\begin{array}{l}\text { A intervenção mostrou-se eficaz na diminuição da } \\
\text { dor e da fadiga }\end{array}$ \\
\hline Hawranik et al. ${ }^{24}$ & $\begin{array}{l}\text { Pessoas com doença de } \\
\text { Alzheimer, apresentando } \\
\text { sintomas de agitação verbal, } \\
\text { agressão física e não física }\end{array}$ & 5 & $\begin{array}{l}\text { Uma vez ao dia durante } \\
5 \text { dias com duração média } \\
\text { de } 30 \text { a } 40 \text { minutos }\end{array}$ & $\begin{array}{l}\text { Os resultados do estudo apresentam um potencial } \\
\text { efeito do Toque Terapêutico na redução dos } \\
\text { sintomas de agitação }\end{array}$ \\
\hline MacIntyre et al..$^{25}$ & $\begin{array}{l}\text { Pessoas submetidas em } \\
\text { procedimento cirúrgico para } \\
\text { revascularização da artéria } \\
\text { coronária }\end{array}$ & 3 & $\begin{array}{l}\text { Uma sessão um dia antes } \\
\text { da cirurgia, uma antes da } \\
\text { cirurgia e uma um dia após a } \\
\text { cirurgia com duração média } \\
\text { de } 20 \text { a } 60 \text { minutos as duas } \\
\text { primeiras sessões e de } 60 \text { a } \\
90 \text { minutos a última sessão }\end{array}$ & $\begin{array}{l}\text { Os resultados do estudo não indicaram melhoras } \\
\text { na diminuição do uso de medicação, diminuição } \\
\text { do tempo de internação, e incidência de fibrilação } \\
\text { cardíaca. Entretanto, ocorreu melhora significativa } \\
\text { nos escores de ansiedade }\end{array}$ \\
\hline Wang et al..$^{26}$ & $\begin{array}{l}\text { Pessoas que sofrem de } \\
\text { demência e apresentam } \\
\text { sintomas de agitação }\end{array}$ & 28 & $\begin{array}{l}\text { Uma vez ao dia durante } \\
4 \text { semanas com duração de } \\
10 \text { minutos cada sessão }\end{array}$ & $\begin{array}{l}\text { Os níveis de agitação foram reduzidos } \\
\text { significativamente com o uso do Toque } \\
\text { Terapêutico }\end{array}$ \\
\hline Movaffaghi et al..$^{16}$ & $\begin{array}{l}\text { Alunos de graduação em } \\
\text { enfermagem com níveis de } \\
\text { hemoglobina inferiores a } \\
12 \text { e } 13 \mathrm{~g} / \mathrm{dl}\end{array}$ & 3 & $\begin{array}{l}\text { Uma sessão a cada dia em } \\
3 \text { dias intervalados com } \\
\text { duração média de } 15 \text { a } 20 \\
\text { minutos }\end{array}$ & $\begin{array}{l}\text { Os resultados sugerem um aumento nos níveis de } \\
\text { hemoglobina e hematócrito }\end{array}$ \\
\hline Larden et al. ${ }^{27}$ & $\begin{array}{l}\text { Mulheres grávidas } \\
\text { em tratamento para } \\
\text { dependência química com } \\
\text { sintomas de ansiedade }\end{array}$ & 7 & $\begin{array}{l}\text { Uma vez ao dia durante } \\
7 \text { dias com duração de } \\
20 \text { minutos cada sessão }\end{array}$ & $\begin{array}{l}\text { Os escores de ansiedade diminuíram } \\
\text { significativamente durante as intervenções com o } \\
\text { Toque Terapêutico }\end{array}$ \\
\hline
\end{tabular}


Os resultados encontrados nesta revisão evidenciam que o uso Toque Terapêutico nas pesquisas tem ocorrido em diferentes situações, com variações no número de sessões aplicadas, no tempo de cada sessão e no intervalo entre as sessões. Por se tratar de um método de intervenção que tem ocasionado o interesse de pesquisadores no mundo todo, caracteriza-se a importância de se estabelecer um consenso em modelos metodológicos que possam ser reproduzidos. ${ }^{15}$

Ressalta-se que, de acordo com o método estabelecido por Krieger, ${ }^{11}$ não existe um número fixo de sessões no qual necessariamente obtenha-se determinado resultado, sendo que o indivíduo deve ser avaliado de acordo com os padrões do seu campo de energia. Entretanto, Hulley et al. ${ }^{28}$ consideram que o uso de determinadas intervenções deva ocorrer quando se tem um amadurecimento suficiente de questões relacionadas à pesquisa. Esses autores observam que, na ausência de evidências significativas sobre determinado tratamento, são recomendados os estudos clínicos para comprovar e validar intervenções.

De acordo com o método Krieger-Kunz, ${ }^{11}$ as sessões de Toque Terapêutico não devem ultrapassar um tempo além de 20 e 25 minutos, em função da sobrecarga energética que pode ser prejudicial ao paciente. Krieger ${ }^{11}$ destaca que a eficiência do tratamento com o Toque Terapêutico não está na maior quantidade de tempo em que se realiza a técnica e, sim, na capacidade do terapeuta em concentrar-se para aplicá-lo adequadamente, tendo sensibilidade para intervir de modo adequado nos diferentes diagnósticos que podem ser encontrados nos CEHs.

Na proposta de intervenção de Toque Terapêutico estabelecida pela Classificação das Intervenções de Enfermagem (NIC) ${ }^{29}$ é notória a falta de definição quanto ao tempo de cada sessão, ao número de sessões, e mesmo às situações em que a técnica pode ser utilizada. Desse modo, vale ressaltar que para o uso desta técnica, tanto nas pesquisas quanto na prática clínica, é fundamental que o terapeuta tenha consciência de que as intervenções devem ocorrer de forma cautelosa, principalmente por considerar que o CEH, bem como o Toque Terapêutico, são universos a serem descobertos.

$\mathrm{Na}$ literatura cientifica, boa parte das pesquisas envolvendo o uso do Toque Terapêutico retrata um tratamento direcionado aos sintomas psicossomáticos. ${ }^{8,9,22,27}$ Krieger $^{11}$ aponta que dentre os sistemas orgânicos mais sensíveis ao Toque Terapêutico, destaca-se o sistema nervoso autônomo.
No entanto, o Toque Terapêutico não se limita a tratar doenças psicossomáticas, e tem sido utilizado de forma complementar no tratamento do câncer, ${ }^{14}$ na cicatrização da pele ${ }^{15}$ e na melhora dos níveis de hemoglobina e hematócrito. ${ }^{16}$

Vasques et al. ${ }^{10}$ observam que nos últimos anos, dentre as terapias complementares mais estudadas, destaca-se o Toque Terapêutico, sendo esta terapia usada principalmente em adultos e idosos com doenças crônicas degenerativas. Tal afirmativa é consonante com os achados desta revisão, em que os estudos se enquadram neste perfil. Compreendem-se as muitas limitações ainda encontradas com o uso do Toque Terapêutico, principalmente relacionados aos protocolos de intervenção. É fundamental que essas limitações sejam superadas, para que no futuro esta modalidade de tratamento possa servir de auxílio no tratamento preventivo de doenças.

Anderson e Taylor ${ }^{30}$ reafirmam as limitações quanto ao uso do Toque Terapêutico, mesmo que em boa parte dos casos ele se mostre benéfico à saúde do indivíduo. Por outro lado, Santos et al. ${ }^{17}$ destacam uma lacuna na descrição dos aspectos fisiológicos encontrados no uso do Toque Terapêutico. Tal afirmativa é consonante com Gerber, ${ }^{12}$ ao afirmar que o maior desafio envolvendo o uso das terapias energéticas é a quantificação dessas intervenções aplicadas.

Considerando as muitas limitações ainda encontradas no uso do Toque Terapêutico, acredita-se que os estudos clínicos de intervenção envolvendo o uso da técnica são fundamentais na busca por explicações sobre os mecanismos envolvidos com o uso da imposição de mãos, principalmente no que se refere ao tempo de cada sessão, número de sessões, intervalo entre as sessões e resultados obtidos. Por outro lado, o estudo realizado por Busch et al. ${ }^{9}$ nos chama atenção para que os estudos científicos de intervenção sejam desenvolvidos por pesquisadores qualificados nesses métodos.

\section{CONSIDERAÇÕES FINAIS}

O Toque Terapêutico é uma terapia complementar que tem despertado o interesse dos pesquisadores nas últimas décadas e vem demonstrando efeitos positivos no seu uso. A intervenção é de baixo custo e, de acordo com a maioria dos estudos, promove benefícios e auxilia o indivíduo no processo de cura. Entretanto, é necessário cautela quanto ao uso da técnica, principalmente em relação aos modelos metodológicos utilizados. 
Pela ausência de uma padronização no seu uso, existem dificuldades na replicação dos modelos já estudados e, consequentemente, na validação de um padrão que possa ser utilizado. Tais dificuldades foram observadas nos resultados deste estudo, que identificou a inexistência de um consenso metodológico relacionado ao tempo, duração e intervalo das sessões realizadas.
Por se tratar de pesquisa secundária, neste estudo observam-se como limitações o processo de seleção dos artigos, em função dos critérios de inclusão estabelecidos e/ou as estratégias adotadas, por impedir que outros estudos possam ser incluídos. No entanto, tal circunstância abre um horizonte que poderá ser continuamente explorado.

\section{REFERÊNCIAS}

1. Cheung CK, Wyman JF, Halcon LL. Use of complementary and alternative therapies in community-dwelling older adults. J Altern Complement Med. 2007 Nov;13(9):997-1006.

2. Shorofi SA, Arbon P. Complementary and alternative medicine (CAM) among hospitalised patients: an Australian study. Complement Ther Clin Pract. 2010 May;16(2):86-91.

3. Bishop FL, Prescott P, Chan YK, Saville J, Von Elm E, Lewith GT. Prevalence of complementary medicine use in pediatric cancer: a systematic review. Pediatrics. 2010 Apr;125(4):768-76.

4. Nahin RL, Barnes PM, Stussman BJ, Bloom B. Costs of Complementary and Alternative Medicine (CAM) and Frequency of Visits to CAM Practitioners: United States, 2007. Hyattsville, MD: National Center for Health Statistics; 2009. National health statistics reports: $\mathrm{n}^{\circ} 18$.

5. Thiago SC, Tesser CD. Percepção de médicos e enfermeiros da estratégia de saúde da família sobre terapias complementares. Rev Saúde Pública. 2011;45(2):249-57.

6. Brasil. Ministério da Saúde. Política Nacional de Medicina Natural e Práticas Complementares PMNPC [Internet]. Brasília; 2005 [cited 2013 Oct 3]. Available from: http://bvsms.saude.gov.br/bvs/publicacoes/ResumoExecutivoMedNatPratCompl1402052.pdf.

7. Woods DL, Craven RF, Whitney J. The effect of therapeutic touch on behavioral symptoms of persons with dementia. Altern Ther Health Med. 2005 Jan-Feb;11(1):66-74.

8. Zolfaghari M, Eybpoosh S, Hazrati M. Effects of therapeutic touch on anxiety, vital signs, and cardiac dysrhythmia in a sample of Iranian women undergoing cardiac catheterization: a quasi-experimental study. J Holist Nurs. 2012 Dec;30(4):225-34

9. Busch M, Visser A, Eybrechts M, Van Komen R, Oen I, Olff M, Dokter J, Boxma H. The implementation and evaluation of therapeutic touch in burn patients: An instructive experience of conducting a scientific study within a non-academic nursing setting. Patient Educ Couns. 2012 Dec;89(3):439-46.

10. Vasques CI, Santos DS, Carvalho EC. Tendências da pesquisa envolvendo o uso do Toque Terapêutico como uma estratégia de enfermagem. Acta Paul Enferm. 2011;24(5):712-4.

11. Krieger D. O Toque Terapêutico: versão moderna da antiga técnica de imposição de mãos. São Paulo: Cultrix; 1995.

12. Gerber R. Medicina vibracional: uma medicina para o futuro. São Paulo: Cultrix; 2007.

13. Brennan BA. Mãos de luz: um guia para a cura através do campo de energia humana. 17ª ed. São Paulo: Pensamento; 1987.

14. Sá AC, Silva MJP. Aplicação do toque terapêutico em mulheres portadoras de câncer de mama sob tratamento quimioterápico. Mundo Saúde. 2003;27(2):258-69.

15. Savieto RM, Silva MJP, Pozzi DHB, Neto PAF. Ação da água energizada com o Toque Terapêutico na cicatrização de lesões na pele de camundongos. Rev Enferm UERJ. 2007;15(3):423-9.

16. Movaffaghi Z, Hasanpoor M, Farsi M, Hooshmand P, Abrishami F. Effects of therapeutic touch on blood hemoglobin and hematocrit level. J Holist Nurs. 2006 Mar;24(1):41-8.Santos DS, Marta IER, Cárnio EC, Quadros AU, Cunha TM, Carvalho EC. Utilização de um modelo experimental para estudo sobre o Toque Terapêutico. Rev Latinoam Enferm. 2013;21(1):1-8.

17. Gomes VM, Silva MJP, Araújo EAC. Efeitos gradativos do Toque Terapêutico na redução da ansiedade de estudantes universitários. Rev Bras Enferm. 2008;61(6):841-6.

18. Moher D, Liberati A, Tetzlaff J, Altman DG; PRISMA Group. Preferred reporting items for systematic reviews and meta-analyses: the PRISMA statement. PLoS Med. 2009 July 21;6(7):e1000097

19. Nascimento SL, Surita FGC, Parpinelli MA, Cecatti JG. Exercício físico no ganho de peso e resultados perinatais em gestantes com sobrepeso e obesidade: uma revisão sistemática de ensaios clínicos. Cad Saúde Pública. 2011;27(3):407-16.

20. Schindel CS, Donadio MVF. Efeitos de programas de exercício físico em pacientes com fibrose cística. Sci Med. 2013;23(3)1-4.

21. Marta IER, Bladan SS, Berton AF, Pavam M, Silva MJP. Efetividade do toque terapêutico sobre a dor, depressão e sono em pacientes com dor crônica: ensaio clínico. Rev Esc Enferm USP. 2010;44(4):1100-6.

22. Aghabati N, Mohammadi E, Pour Esmaiel Z. The effect of therapeutic touch on pain and fatigue of cancer patients undergoing chemotherapy. Evid Based Complement Alternat Med. 2010 Sept;7(3):375-81.

23. Hawranik P, Johnston P, Deatrich J. Therapeutic touch and agitation in individuals with Alzheimer's disease. West J Nurs Res. 2008 June;30(4):417-34. 
24. MacIntyre B, Hamilton J, Fricke T, Ma W, Mehle S, Michel M. The efficacy of healing touch in coronary artery bypass surgery recovery: a randomized clinical trial. Altern Ther Health Med. 2008 July-Aug;14(4):24-32.

25. Wang KL, Hermann C. Pilot study to test the effectiveness of Healing Touch on agitation in people with dementia. Geriatr Nurs. 2006 Jan-Feb;27(1):34-40.

26. Larden CN, Palmer ML, Janssen P. Efficacy of therapeutic touch in treating pregnant inpatients who have a chemical dependency. J Holist Nurs. 2004 Dec;22(4):320-32.

27. Hulley SB, Cummings SR, Browner WS, Grady DH, Newman TB. Delineando a pesquisa clinica: uma abordagem epidemiológica. $3^{3}$ - ed. Porto Alegre: Artmed; 2008.

28. Bulechek GM, Butcher HK, Dochterman JM. Classificação das intervenções de enfermagem (NIC). 5aㅡ. ed. Rio de Janeiro: Elsevier; 2010.

29. Anderson JG, Taylor AG. Effects of healing touch in clinical practice: a systematic review of randomized clinical trials. J Holist Nurs. 2011 Sept;29(3):221-8. 